\title{
Levels of extra domain A containing fibronectin in human atherosclerotic plaques are associated with a stable plaque phenotype
}

\author{
J. Karlijn van Keulen ${ }^{a, b}$, Dominique P. de Kleijn ${ }^{a, b}$, Manon M. Oude Nijhuis ${ }^{a, b}$, \\ Els Busser ${ }^{\mathrm{a}}$, Evelyn Velema ${ }^{\mathrm{a}}$, Rob Fijnheer ${ }^{\mathrm{c}}$, Yolanda van der Graaf ${ }^{\mathrm{d}}$, \\ Frans L. Moll ${ }^{\mathrm{e}}$, Jean Paul de Vries ${ }^{\mathrm{f}}$, Gerard Pasterkamp ${ }^{\mathrm{a}, *}$ \\ a Experimental Cardiology Laboratory, Cardiology, University Medical Center Utrecht, The Netherlands \\ ${ }^{\mathrm{b}}$ Interuniversity Cardiology Institute of the Netherlands (ICIN), Utrecht, The Netherlands \\ ${ }^{\mathrm{c}}$ Haematology, University Medical Center Utrecht, The Netherlands \\ d Julius Center for Health Sciences and Primary Care, University Medical Center Utrecht, The Netherlands \\ e Vascular Surgery, University Medical Center Utrecht, The Netherlands \\ ${ }^{\mathrm{f}}$ Vascular Surgery, Antonius Hospital Nieuwegein, The Netherlands
}

Received 30 November 2006; received in revised form 2 March 2007; accepted 8 March 2007

Available online 9 April 2007

\begin{abstract}
Background: Extra domain A (EDA), splice-variant of fibronectin, is a Toll-like receptor 4 (Tlr4) ligand. Recently, EDA has been demonstrated to enhance atherogenesis in mice but human data on the role of EDA in atherosclerotic disease are lacking. We hypothesized that EDA is associated with unstable plaque phenotypes and that plasma EDA could serve as biomarker for atherosclerosis.

Methods: EDA levels were assessed in carotid endarterectomy specimen (206 patients) and related with plaque phenotype. In a second patient cohort, systemic EDA levels in atherosclerotic patients (73 patients) were compared to risk-factor matched controls (68 patients).

Results: EDA plaque levels were associated with characteristics of stable plaques; more smooth muscle cells $(P=0.003)$, more collagen $(P=0.071)$ and less fat $(P=0.023)$. Concomitantly, asymptomatic patients showed higher EDA values in the plaque compared to symptomatic patients $(P=0.004)$. EDA plasma levels did not differ between atherosclerotic patients versus controls $(P=0.134)$.

Conclusion: EDA plaque levels are higher in asymptomatic patients and are associated with a stable plaque phenotype. EDA is not a plasma marker for atherosclerotic disease. These results suggest that local presence of endogenous Tlr4 ligand EDA is not associated with in an unstable plaque phenotype in humans.
\end{abstract}

(C) 2007 Elsevier Ireland Ltd. All rights reserved.

Keywords: Atherosclerosis; Plaque; Fibronectin; Extra domain A

\section{Introduction}

Fibronectin is a major component of the extracellular matrix (ECM). Fibronectins are high molecular weight glycoproteins, that play key roles in cell adhesion, migration, growth and differentiation [1]. Alternative splicing of the pri-

\footnotetext{
* Corresponding author at: Experimental Cardiology Laboratory, Heidelberglaan 100, Room G02-523, 3584 CX Utrecht, The Netherlands. Tel.: +31 302507155; fax: +31 302522693 .

E-mail address: g.pasterkamp@umcutrecht.nl (G. Pasterkamp).
}

mary transcript of the fibronectin gene results in the insertion or exclusion of certain domains within the fibronectin protein [2]. Extra domain A (EDA or EIIIA) is a fibronectin domain that is included by alternative splicing during embryogenesis, wound healing and cellular damage [2,3].

Expression of fibronectin containing EDA is increased in pathophysiological conditions, for example, in graft versus host disease leading to fibrosis of the skin, fibrosis in lung, liver and kidney, diabetes, rheumatoid arthritis, cardiac transplantation, vascular intimal proliferation and vascular hypertension [4-13]. 
EDA is known to activate Toll-like receptor 4 (Tlr4) [14]. Tlrs are the first line of defense in the innate immune response following recognition of pathogen-associated molecular patterns. To date, 10 human Tlrs have been identified. Tlr4 recognizes exogenous ligands, like bacterial lipopolysaccharide, but also endogenous ligands, like EDA and heat shock protein 60 [14-16].

The presence of Tlr4 has been documented in murine and human atherosclerosis [17]. Recently, we and others showed that Tlr4 activation accelerates arterial occlusion by neointima formation, plaque formation, geometrical remodeling and plaque destabilization [18-21]. It remains unclear which ligands activate Tlr4 and thereby promote atherosclerotic luminal narrowing. Exogenous ligands are not obligatory for development of murine atherosclerosis [22] and are not involved in Tlr4 dependent flow induced expansive remodeling [18].

The endogenous Tlr4 ligand EDA is expressed in human and murine atherosclerotic lesions [23]. Evidence that EDA may act as a trigger for atherogenesis is accumulating. EDA levels are elevated in plasma of ApoE knockout mice [23] and EDA mRNA levels are increased during Tlr4-dependent outward remodeling [18]. Furthermore, in atherosclerotic mice lacking EDA, atherosclerotic lesion areas were found to be reduced in number and size [23].

Human studies in which EDA levels are studied in atherosclerotic plaques and plasma in relation with clinical presentation are lacking. We hypothesized that local EDA levels in the atherosclerotic plaque are associated with a more inflammatory and advanced stage of atherosclerotic disease. We therefore determined EDA levels in human atherosclerotic plaques and plasma samples obtained in two patient cohorts to assess associations with plaque characteristics and the presence of clinically manifest atherosclerotic disease.

We report that EDA levels in the plaque are associated with a stable plaque phenotype. No association was observed between plasma EDA and the presence of clinically manifest atherosclerotic disease. Our results suggest that EDA is not a serological biomarker for atherosclerotic disease but that plaque EDA could serve as a potential local marker associated with a stable plaque phenotype.

\section{Materials and methods}

\subsection{Clinical studies}

\subsubsection{Athero-Express}

Athero-Express is an ongoing longitudinal cohort study, initiated in 2002 by two Dutch hospitals [24]. The study has been approved by the ethics committees of both hospitals and written informed consents were obtained from all participants. The study is designed to investigate the expression of atherosclerotic tissue derived biological markers in relation to plaque phenotype and the long-term outcome of patients undergoing carotid endarterectomy (CEA). Patients who undergo CEA fill in an extensive questionnaire and diagnostic examinations are performed. The Athero-Express biobank furthermore contains blood samples drawn before surgery and freshly frozen atherosclerotic tissue to study protein and RNA expression. We used plaque samples from the Athero-Express study to answer the research question whether EDA plaque levels are associated with stable or unstable plaques.

\subsubsection{SMART}

The Second Manifestations of ARTerial disease (SMART) study is an ongoing, single-center, prospective cohort study of approximately 3500 patients. The patients are referred to the University Medical Center Utrecht for the first time because of atherosclerotic vascular disease or treatment of atherosclerotic risk factors [25]. The study was approved by the ethics committee of the University Medical Center Utrecht, and written informed consent was obtained from all participants.

For the present study, patients were selected that fulfilled the following three criteria: (1) a history of clinically manifest atherosclerotic disease (referral for percutaneus peripheral or coronary intervention, surgery to treat aneurysm formation, $\mathrm{CABG}$, surgery to treat peripheral arterial disease, stroke); (2) atherosclerotic disease at the time of hospital-entry and (3) one cardiovascular risk factor (hypertension, diabetes mellitus or hyperlipidemia). All patients had been screened for the presence of clinically silent atherosclerotic lesions in the carotid and femoral arteries and aorta using three approaches. First, atherosclerotic luminal narrowing in the femoral artery was evaluated by measuring the ratio of the systolic blood pressure measured at the ankle to the systolic blood pressure measured in both arms (ankle-brachial pressure index, ABPI). When patients without symptoms had a lowered ABPI $(\leq 0.9)$ they were classified as having silent atherosclerosis. Second, stenosis of the common and internal carotid arteries was measured bilaterally with Doppler-assisted duplex scanning. When carotid stenosis exceeded $70 \%$, patients were diagnosed having clinically silent atherosclerosis. Third, ultrasonography of the abdomen was performed to measure the anteroposterior juxtarenal diameter and the distal anteroposterior diameter of the aorta. When distal anteroposterior diameter $\geq 3 \mathrm{~cm}$ or $\geq 1.5$ times anteroposterior juxtarenal diameter, patients were diagnosed having clinically silent atherosclerosis.

Control patients from the SMART study were frequencymatched for sex, age and one of three major risk factors (hypertension, diabetes mellitus and hyperlipidemia). These patients revealed neither clinically evident nor silent atherosclerosis.

The SMART cohort was used to answer the research question whether EDA could serve as a plasma biomarker for the presence of atherosclerotic disease.

\subsubsection{Handling of atherosclerotic specimens}

Atherosclerotic specimens obtained by carotid endarterectomy were dissected in segments of $0.5 \mathrm{~cm}$ in length. The 
segment with the largest plaque size (numbered 0) was fixated in formaldehyde $4 \%$ and paraffin embedded. The adjacent segments (numbered + or $-1,2,3$, etc.) were immediately frozen in liquid nitrogen and stored at $-80^{\circ} \mathrm{C}$. Paraffin sections of the atherosclerotic plaques were immunohistochemically stained and scored for the presence of macrophages (CD68), smooth muscle cells (alpha actin), collagen (picro Sirius red) and fat. Collagen staining was determined using polarized light microscopy on picro Sirius red stainings and scored as (1) no or minor staining = staining along part of the luminal border; (2) moderate or heavy staining $=$ staining along the entire luminal border. The percentage atheroma of the total area of the plaque is visually estimated using haematoxylin stains. Three groups were considered based on the percentage of atheroma in the plaque: fibrous plaques containing $<10 \%$ fat; fibro-atheromatous, $10-40 \%$; or atheromatous, $>40 \%$ fat. The CD68 (macrophage) and smooth muscle alpha actin staining were analyzed quantitatively by computerized analyses using Analysis ${ }^{\circledR}$ software. Color thresholds were set and adjusted until the computerized detection met the visual interpretation. The stained areas were scored quantitatively as a percentage of the plaque area. All semiquantitative scores were performed independently by two observers.

Proteins were isolated from the adjacent (+1) segments using $1 \mathrm{~mL}$ Tripure ${ }^{\mathrm{TM}}$ Isolation Reagent (Boehringer, Mannheim) according to the manufacturer's protocol.

\subsubsection{Mammary arteries}

Mammary arteries were used as non-atherosclerotic control arteries. Segments of mammary arteries $(n=6)$ were obtained during bypass surgery. Levels of EDA protein in the mammary arteries were compared to nine Athero-Express protein samples, measured all together on one ELISA plate.

\subsubsection{Immunohistochemistry}

Sections of atherosclerotic lesions were immunohistochemically stained for EDA (IST-9, Abcam, Cambridge), $\mathrm{IgG}_{1}$ as isotype control, macrophages (CD68), SMCs (alphaactin) and endothelial cells (CD31).

EDA and $\mathrm{IgG}_{1}$ stainings were developed with fast blue, macrophages with fast red, the endothelial cells and SMCs with AEC. Haematoxylin was used for counterstaining.

\subsubsection{EDA enzyme-linked immuno sorbent assay}

Fibronectin containing EDA was measured with an enzyme-linked immunosorbent assay (ELISA) developed at the University Medical Center Utrecht [8]. Microtiter plates were coated overnight with $\mathrm{IgG}_{1}$ monoclonal antibody IST-9 $(1.06 \mu \mathrm{g} / \mathrm{mL})$ directed against Extra domain A of fibronectin (Abcam, Cambridge, UK) in carbonate buffer $\left(\mathrm{Na}_{2} \mathrm{CO}_{3} 15 \mathrm{mM}, \mathrm{NaHCO}_{3} 35 \mathrm{mM}, \mathrm{pH} 9.6\right)$ at $4{ }^{\circ} \mathrm{C}$. Wells were washed with PBS/0.1\% Tween and then blocked with phosphate-buffered saline (PBS)/3\% bovine serum albumin (BSA) $/ 0.1 \%$ Tween. Samples were diluted to an appropriate concentration with PBS/0.1\% Tween/3\% BSA (1:20 for plasma and 1:100 for protein extracts in 1\% SDS). Ligand capture was detected by addition of peroxidase-conjugated rabbit-anti-human fibronectin antibodies $(1.3 \mu \mathrm{g} / \mathrm{mL})$ (Dako, Glostrup, Denmark). Binding of the antibody was detected by adding $1 \mu \mathrm{g} / \mathrm{mL} O$-phenylene-diamine in $0.05 \mathrm{M}$ citratephosphate buffer ( $\mathrm{pH} 5$ ) with $2.5 \mathrm{mM} \mathrm{H}_{2} \mathrm{O}_{2}$. The reaction was stopped by adding $1 \mathrm{M}$ sulfuric acid. Absorbance was read at $490 \mathrm{~nm}$ on microplate reader model 550, Biorad, $655 \mathrm{~nm}$ filter was used as reference-filter. As a standard, cellular fibronectin was purified from cultured human fetal lung fibroblasts [26]. EDA levels measured by ELISA were corrected for protein concentrations of the samples.

\subsubsection{MMP, IL and Emmprin measurements in plaques}

MMP-2, MMP-9 are most intensively studied MMPs in relation to atherosclerosis and have been associated with matrix degradation in unstable lesions. MMP-8 has been related with thrombus formation. Recently we demonstrated an increase of MMP-8 and MMP-9 activity levels in unstable plaques, whereas MMP-2 activity levels are higher in stable lesions [27].

In 119 protein samples from a randomly selected subgroup of the Athero-Express study, matrix metalloproteinase (MMP)-2, MMP-8 and MMP-9 activities were measured using the Biotrak activity assays RPN 2631, RPN 2635 and RPN 2634 (Amersham Biosciences), respectively.

Extracellular matrix metalloproteinase inducer (Emmprin) expression levels were determined by Western blotting [27] (sc-9753, Santa Cruz Biotechnology).

Interleukin (IL)-6 and IL-8 are pro-inflammatory cytokines that have been associated with enhanced inflammatory response in atherosclerotic lesions. We measured IL-6 and IL-8 levels by a multiplex suspension array system according to the manufacturer's protocol (Bio-Rad Laboratories).

\subsubsection{Statistics}

The Mann-Whitney $U$ or Kruskal-Wallis test was performed to compare different variables. Linear regression analyses were performed to correlate plasma and plaque EDA. A $P<0.05$ was regarded significant.

\section{Results}

\subsection{Baseline characteristics of Athero-Express and SMART study population}

The baseline characteristics of the Athero-Express and SMART study population are presented as Supplementary data. Besides the difference in intima media thickness as measure of extent of atherosclerotic disease, which is higher in atherosclerotic patients, the cholesterol levels in atherosclerotic patients are lower than in the controls. This is probably the result of statin treatments the atherosclerotic patients received. No differences were observed in the other baseline 
characteristics (age, gender, body mass index, hypertension, hyperlipidemia, diabetes mellitus, smoking, cholesterol, triglycerides, HDL-cholesterol, glucose) between atherosclerotic patients and controls in the SMART study population.

\subsection{EDA levels in the atherosclerotic plaque}

\subsubsection{EDA expression levels in plaques compared to non-atherosclerotic arteries}

Atherosclerotic plaques revealed higher EDA levels compared to non-atherosclerotic mammary arteries $(n=9$ and $n=6$, respectively, $22.9 \pm 5.5 \mu \mathrm{g} / \mathrm{mL}$ versus $6.8 \pm$ $1.7 \mu \mathrm{g} / \mathrm{mL}, P=0.003$, Supplementary data, Fig. 1). Mammary arteries did not show EDA staining, confirming the difference in EDA expression measured by ELISA (Supplementary data, Fig. 1b-e). To determine which cells do express EDA in the plaque, sections were immunohistochemically stained for EDA. EDA expression was predominantly observed in the extracellular matrix around macrophages, but also in SMC-rich areas and around endothelial cells (Fig. 1).

\subsubsection{EDA levels and plaque phenotype}

Atheromatous plaques $(n=63)$ showed significantly lower EDA values compared to fibrous $(n=67)$ and fibroatheromatous plaques $(n=72),(15.5 \pm 3.0,19.4 \pm 2.3$ and $20.8 \pm 2.2 \mu \mathrm{g} / \mathrm{mL}$, respectively, $P=0.023$, Fig. 2a).

Fibrous plaques are commonly associated with strong collagen and smooth muscle cell staining, while the atheromatous plaque is associated with an inflammatory unstable plaque phenotype. Fig. $2 \mathrm{~b}$ and $\mathrm{c}$ show that plaques with higher EDA expression, contain more collagen (although not significantly different, there is a strong trend towards more collagen in plaques with more EDA in the plaque) and more smooth muscle cells (Fig. 2b and c, $P=0.071$ and $P=0.003$, respectively). The lower percentage of SMC of plaques in quartile 1, expressing the lowest EDA levels, differs from the SMC percentages of the plaques in the other quartiles. No statistical relation is observed between EDA levels and the inflammatory macrophage cell content (Fig. 2d, $P=0.517$ ). Smooth muscle cells mostly outnumber the macrophages. This might obscure a possible relation between macrophage presence and EDA. Therefore, we assessed the relation of macrophages and EDA expression in the plaques that contained low
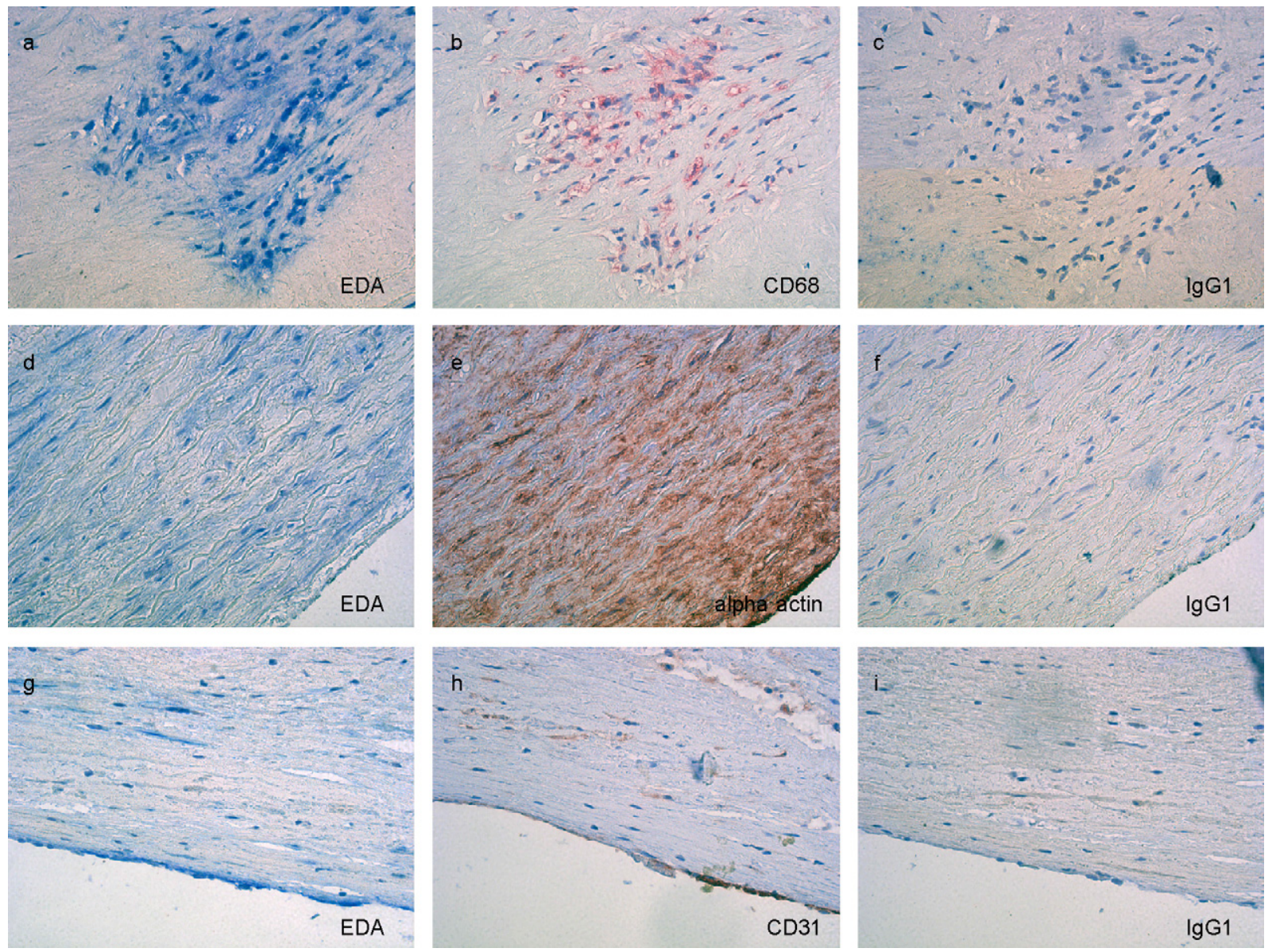

Fig. 1. EDA expression in atherosclerotic lesions. EDA staining ( $\mathrm{a}, \mathrm{d}, \mathrm{g})$ is predominantly co-localized with staining for macrophages (CD68, b), but also observed in smooth muscle cell rich areas (alpha actin, e) and around endothelial cells (CD31, h). IgG controls are negative (c, f, i). All pictures are taken with $400 \times$ magnification. 

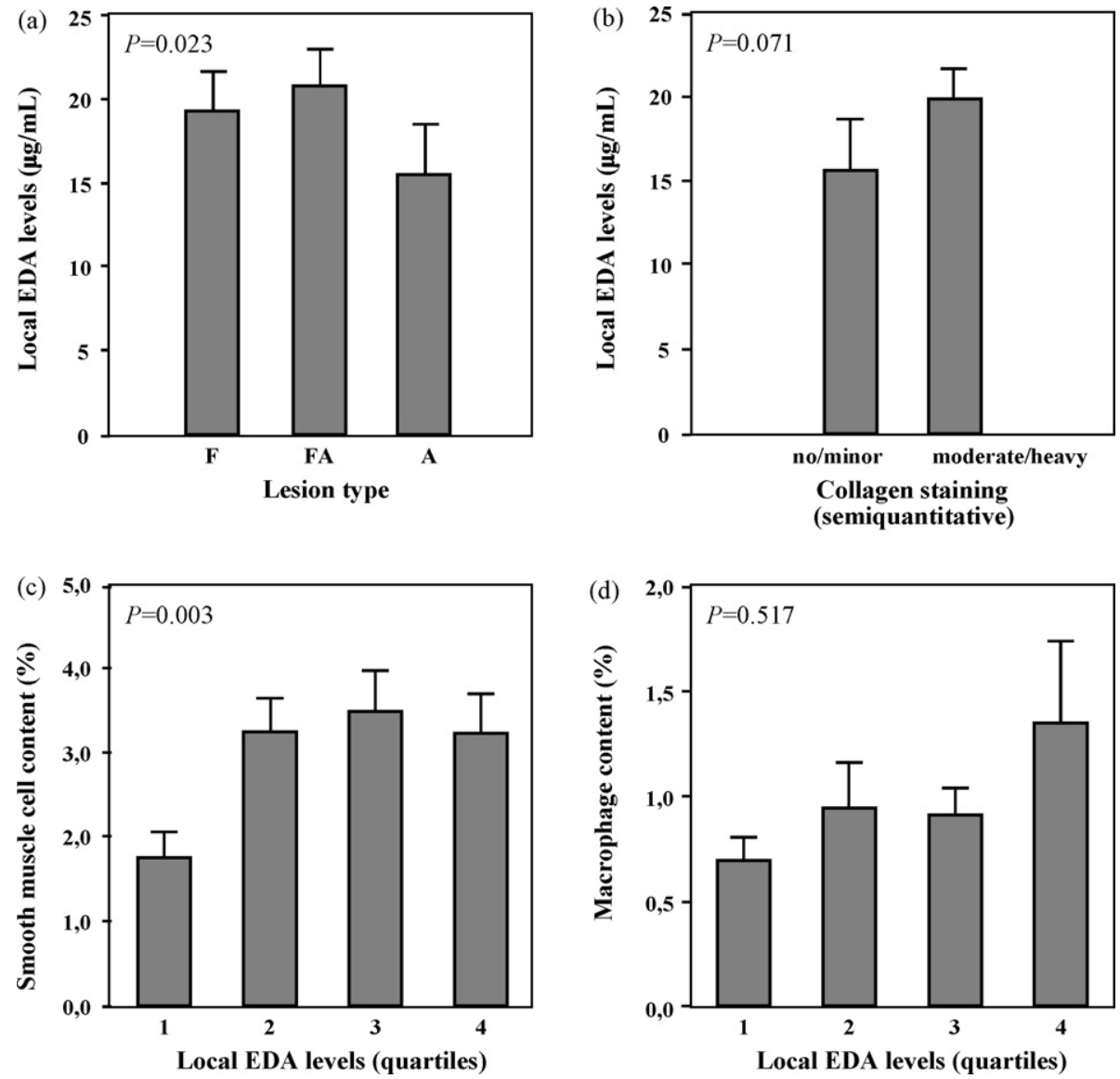

Fig. 2. (a-d) Relation of local EDA levels with amount of collagen, fat, macrophages and smooth muscle cells in the plaque. The plaques were divided into three groups; fibrous (F, $n=67$ ), fibroatheromatous (FA, $n=72)$ and atheromatous (A, $n=63)$, based on fat content $(<10 \%,<40 \%$ or $>40 \%$ fat, respectively). Higher local EDA levels were detected in the fibrous plaques (a, $P=0.023)$. EDA levels in plaques with no/minor collagen staining $(n=52)$ are compared to plaques with moderate/heavy collagen staining $(n=147)$. Local EDA levels are associated with more collagen content $(\mathrm{b}, P=0.071)$ and a higher number of smooth muscle cells in the plaque (c, $P=0.003)$. No relation is found between the number of macrophages in the plaque and local EDA levels $(\mathrm{d}, P=0.517)$. Error bars in (a-d) represent the standard error of the mean (S.E.M.).

numbers of smooth muscle cells. Also in this group no relation between EDA levels and macrophage content was observed in the plaques (data not shown).

In the atherosclerotic plaque samples the expression levels of MMPs, MMP inducer Emmprin, IL-6 and IL-8 were determined (Table 1). Emmprin is expressed in two glycosylated forms in plaques; 45 and $58 \mathrm{kDa}$. Previously, smooth muscle cell rich, stable lesions were associated with high Emmprin $45 \mathrm{kDa}$ and MMP-2 levels. In contrast, unstable inflammatory lesions were associated with Emmprin $58 \mathrm{kDa}$ and MMP-9 levels [27]. The expression of $45 \mathrm{kDa}$ variant of MMP inducer Emmprin was positively correlated with EDA in the plaque, while the $58 \mathrm{kDa}$ variant showed a trend to correlation with EDA ( $45 \mathrm{kDa}, P<0.001 ; 58 \mathrm{kDa}, P=0.072$ ). In Table 1 , the EDA levels in $\mu \mathrm{g} / \mathrm{mL}$ ( \pm S.E.M.) are shown for the different components divided into quartiles. EDA expression increased when more MMP-2 was expressed in the plaque $(P=0.001)$. No association between EDA expression levels and MMP-8 and -9 was observed in the plaques $(P=0.929$ and $P=0.121)$. A low ratio of Emmprin $45 / 58 \mathrm{kDa}$ is an indicator of the activity status of Emmprin. This ratio was positively associated with higher EDA expression levels $(P=0.010)$. We observed no relation between IL-6 and EDA levels $(P=0.138)$. IL-8 levels showed an inverse relation with EDA expression in the plaques $(P=0.002)$.

\subsubsection{EDA levels in symptomatic versus asymptomatic subjects}

Verhoeven et al. [28] reported that patients with vulnerable plaque phenotypes more often suffered from symptoms related with cerebral ischemia compared to patients with stable plaques. We investigated whether plaques obtained from symptomatic versus asymptomatic showed different EDA expression levels. Patients undergoing carotid endarterectomy who had suffered from any clinical symptom related to carotid atherosclerosis, like TIA, stroke or amaurosis fugax, were considered symptomatic. Concomitantly, patients who did not suffer from clinical symptoms were asymptomatic: their indication for surgical therapy depended on co-morbidity and vertebral-basilar (in)sufficiency. 
Table 1

Plaque EDA levels in $\mu \mathrm{g} / \mathrm{mL}$ ( \pm S.E.M.) in quartiles of inflammatory components

\begin{tabular}{|c|c|c|c|c|c|}
\hline & \multicolumn{4}{|l|}{ Quartiles } & \multirow[t]{2}{*}{$P$-value } \\
\hline & 1 & 2 & 3 & 4 & \\
\hline MMP-2 & $9.6( \pm 1.8)$ & $17.3( \pm 2.6)$ & $19.3( \pm 3.1)$ & $29.3( \pm 4.6)$ & 0.001 \\
\hline MMP-9 & $20.4( \pm 2.3)$ & $18.2( \pm 2.7)$ & $24.7( \pm 5.2)$ & $12.4( \pm 2.2)$ & 0.121 \\
\hline MMP-8 & $18.0( \pm 2.9)$ & $16.2( \pm 2.4)$ & $20.7( \pm 3.6)$ & $20.8( \pm 4.5)$ & 0.929 \\
\hline Emmprin $45 \mathrm{kDa}$ & $10.5( \pm 3.6)$ & $17.3( \pm 2.9)$ & $22.2( \pm 3.2)$ & $24.8( \pm 3.2)$ & $<0.001$ \\
\hline Emmprin $58 \mathrm{kDa}$ & $14.1( \pm 3.5)$ & $21.8( \pm 2.9)$ & $17.6( \pm 2.2)$ & $21.4( \pm 4.3)$ & 0.072 \\
\hline Ratio emmprin $45 / 58 \mathrm{kDa}$ & $13.6( \pm 3.6)$ & $18.7( \pm 3.9)$ & $17.9( \pm 2.3)$ & $26.5( \pm 3.6)$ & 0.010 \\
\hline IL-6 & $25.0( \pm 4.0)$ & $14.1( \pm 2.0)$ & $16.3( \pm 2.9)$ & $19.0( \pm 3.1)$ & 0.138 \\
\hline IL-8 & $24.0( \pm 3.1)$ & $19.9( \pm 3.5)$ & $19.3( \pm 3.2)$ & $11.2( \pm 2.3)$ & 0.002 \\
\hline
\end{tabular}

We also determined the correlation (Spearman test) between EDA expression levels and the levels of inflammatory components. MMP2, rho $=0.377, P=<0.001$; MMP9, rho $=-0.222, P=0.015 ;$ MMP8, rho $=-0.031, P=0.740 ;$ Emmprin $45 \mathrm{kDa}$, rho $=0.424, P=<0.001 ;$ Emmprin $58 \mathrm{kDa}$, rho $=0.137, P=0.131 ;$ Ratio emmprin 45/58 kDa, rho $=0.329, P=<0.001$; IL-6, rho $=-0.082, P=0.253$; IL-8, rho $=-0.257, P=<0.001$.

Higher EDA plaque values were found in asymptomatic subjects compared to symptomatic subjects $(27.0 \pm 3.8$ $\mu \mathrm{g} / \mathrm{mL}$ versus $17.1 \pm 1.6 \mu \mathrm{g} / \mathrm{mL}, P=0.004$, Fig. 3 ).

\subsection{Systemic EDA levels}

\subsubsection{EDA levels in plasma from atherosclerotic patients and controls (SMART)}

Having established that EDA levels are increased in atherosclerotic specimen compared with non-atherosclerotic arteries, we investigated whether systemic EDA levels in plasma were related with clinical manifestation of atherosclerotic disease.

EDA levels were measured with ELISA in plasma of 73 atherosclerotic patients and 68 risk-factor matched controls. All control patients scored negative when screened for the presence of clinically silent peripheral atherosclerotic disease (see Section 2). Plasma EDA levels did not

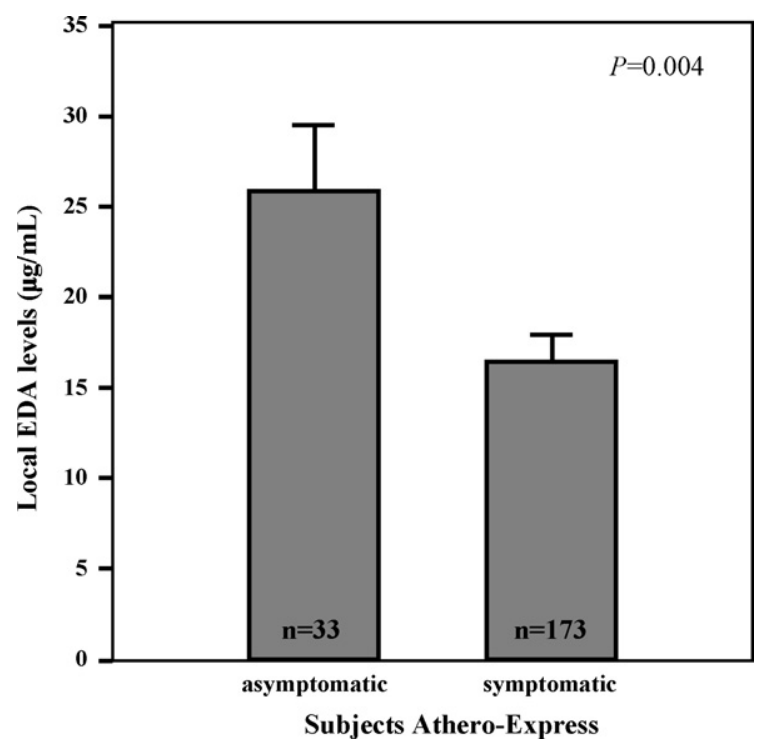

Fig. 3. EDA levels in plaques from asymptomatic versus symptomatic subjects from the Athero-Express study. Asymptomatic patients show higher EDA plaque values compared to symptomatic patients $(27.0 \pm 3.8 \mu \mathrm{g} / \mathrm{mL}$ versus $17.1 \pm 1.6 \mu \mathrm{g} / \mathrm{mL}, P=0.004)$. Error bars represent S.E.M. differ between patients and controls $(6.4 \pm 0.5 \mu \mathrm{g} / \mathrm{mL}$ versus $7.6 \pm 0.6 \mu \mathrm{g} / \mathrm{mL}$, respectively, $P=0.134$, Fig. $4 \mathrm{a}$ ).

All patients in the SMART cohort underwent duplex ultrasound measurements to assess carotid intima media thickness. SMART subjects were divided in quartiles based on their measured carotid intima-media thickness, which is representative for the severity of atherosclerotic disease. Although a tendency was suspected towards an inverse relation between intima media thickness and EDA levels, no significant difference was found in EDA plasma levels between intima media thickness quartiles $(P=0.182$, Fig. $4 \mathrm{~b})$.

\section{Discussion}

Extra domain A is incorporated in fibronectin during embryogenesis and repair processes after tissue-injury. In this study, we investigated whether human EDA levels local in the plaque and systemic in plasma could be associated with plaque characteristics and used as marker for atherosclerotic disease.

\subsection{Local EDA levels in the plaque}

In atherosclerotic lesions, EDA expression levels were higher compared to non-atherosclerotic mammary arteries. Different cell types in the atherosclerotic lesions have been co-localized with EDA expression, like endothelial cells, macrophages and smooth muscle cells [2,12,23,29].

Local EDA levels were associated with a more stable plaque phenotype. Fibrous and fibroatheromatous lesions express higher EDA levels compared to atheromatous plaques. Furthermore, EDA expression is associated with more collagen and more smooth muscle cells, characteristic for a stable plaque phenotype. Concomitantly, higher EDA expression levels were observed in asymptomatic patients compared to patients with clinical symptoms related to atherosclerosis. This is in accordance with Verhoeven et al. [28], who showed that patients with vulnerable plaque phenotypes exhibit more symptoms related to atherosclerosis than patients with stable plaque phenotypes. 

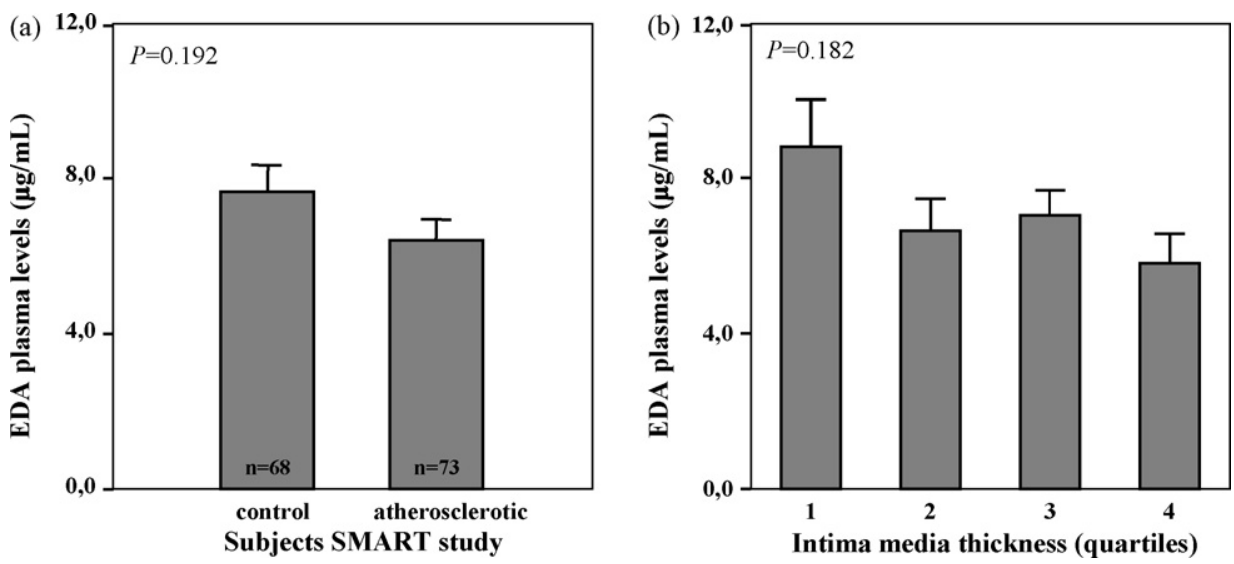

Fig. 4. EDA levels in plasma in $\mu \mathrm{g} / \mathrm{mL}$ of atherosclerotic patients $(n=73)$ and risk-factor matched controls $(n=68)$ from the SMART study. No difference is found between the patient and control-group $(7.1 \pm 0.7 \mu \mathrm{g} / \mathrm{mL}$ versus $6.9 \pm 0.5 \mu \mathrm{g} / \mathrm{mL}$, respectively, $P=0.192$ (a). Error bars represent S.E.M. No difference in EDA levels was found in the SMART population divided into quartiles based on intima-media thickness $(8.8 \pm 1.2 \mu \mathrm{g} / \mathrm{mL} ; 6,6 \pm 0.8 \mu \mathrm{g} / \mathrm{mL} ; 7.0 \pm 0.6 \mu \mathrm{g} / \mathrm{mL}$; $5.8 \pm 0.8 \mu \mathrm{g} / \mathrm{mL}, P=0.182$ (b). Error bars represent S.E.M.

The finding that local EDA levels are higher in atherosclerotic specimen compared to a healthy vessel wall and considering the association of EDA with plaque phenotypes, suggests a role for EDA in atherosclerosis. The dual role of EDA in fibronectin as major component of the ECM and as activator of the immune system via Tlr4 [14] make EDA a good candidate to be involved in the initiation and progression of atherosclerosis, in which both inflammation and matrix turn-over are important processes.

As a ligand of Tlr4 [14] and with atherosclerotic EDA null mice having less atherosclerosis and a more stable plaque phenotype [23], we expected a correlation between high EDA plaque levels and a more inflammatory and unstable plaque phenotype. However, in plaques with atheromatous phenotypes the EDA levels were found to be lower and EDA levels were not associated with the presence of macrophages. These surprising observations might be explained by the possible differences in the mechanisms for atherosclerotic lesion development between mice and humans. In the present study, atherosclerotic lesions have been investigated that resulted in a clinical syndrome, which is not observed in genetically modified atherosclerotic mice. In these lesions high EDA levels were related with stable, non-inflammatory plaques. This observation does not exclude an inflammatory role for EDA during the initiation and early progression of the atherosclerotic plaque as shown in EDA KO mice [23], which points to EDA as a key molecule in the initiation phase of atherosclerosis.

Damage to arterial wall cells, for example, to the endothelium, is one of the first triggers to atherosclerosis. The response of the arterial wall to damage is scarring, which includes ECM formation, SMC proliferation and also EDA production as shown by Dubin et al. [11]. EDA in the plaque may activate Tlr4 and initiate an inflammatory response, like a fraction of EDA is able to activate Tlr4 and initiate an inflammatory cascade [14]. But Tlr4 activation is also important for collagen turnover [20] and VSMC proliferation [30]. EDA might trigger SMC proliferation via Tlr4 activation, leading to stabilization and reorganization of the plaque, which results in conversion of an unstable inflammatory plaque into a stable, smooth muscle cell rich plaque. Tlr4 expression has been described in macrophages in murine and human atherosclerotic lesions [17,31]. Immunohistochemistry showed EDA-staining around macrophages in the lesions, however, no association was observed between the number of plaque macrophages and plaque EDA levels. In contrast to smooth muscle cells, the more SMC, the more EDA is measured in the plaque, suggesting that not macrophages but smooth muscle cells are the dominant EDA producing cells in the human atherosclerotic plaque. Glukhova et al. [12] demonstrated that EDA is expressed in the intima of human and experimental animal arteries by SMC that underwent a phenotype switch; from contractile to synthetic/fibroblast-like SMCs. Synthetic SMCs do have a high proliferation and migration rate and produce extra cellular matrix components. These capacities are important during the initiation of atherosclerosis when SMCs migrate from the medial layer into the intima, where they will proliferate and produce extracellular matrix components. In more advanced atherosclerotic lesions these synthetic SMCs may have a different role. These smooth muscle cells produce next to fibronectin containing EDA [2,3] also collagen [32] and other matrix molecules for repair of the arterial wall. This is in agreement with the previously observed correlations between EDA, collagen content, MMP2 and Emmprin $45 \mathrm{kDa}$ levels [27] in the plaque. SMC proliferation and their production of ECM components may stabilize or reorganize the plaques from unstable into more stable atherosclerotic lesions.

The absence or even inverse relation between EDA and IL-8, IL-6, MMP-8 and MMP-9 supports the idea that local inflammation is not associated with local EDA levels. Tendencies towards inverse associations between the presence of macrophages, IL-8 and EDA were observed. Therefore, this 
study is not likely underpowered to support the hypothesis of a positive association between local inflammation and EDA.

\subsection{EDA levels in plasma}

Although plaque EDA levels are associated with plaque phenotype, EDA plasma levels did not correlate with the severity of atherosclerosis, measured as the intima-media thickness in the SMART population. In addition, EDA levels did not differ between atherosclerotic patients and risk factor-matched controls.

EDA is a high molecular weight protein associated with the extracellular matrix. This may explain why EDA is not released from the arterial wall during atherosclerosis. However, in diabetic patients EDA is released from the vessel wall into the circulation and EDA levels are associated with diabetes [8]. EDA is involved in many other processes and disorders related to pathophysiological circumstances in the vessel wall [4-13]. EDA levels in plasma may also represent other pathological or physiological changes and might mask the changes due to atherosclerosis. Furthermore, also processing of EDA into smaller fragments might be necessary to get an immune reaction. These are two possible explanations why we did not find a difference in EDA containing fibronectin levels between atherosclerotic subjects and risk factor matched controls. In Athero-Express plasma samples EDA levels were also assessed. The systemic levels did not correlate with EDA plaque levels, or with plaque phenotype (data not shown), which indicates that plasma EDA levels are not useful as marker for atherosclerosis.

\subsection{Limitations of the study}

Internal mammary arteries have been used as control arteries, since they are spared from atherosclerosis possibly due to biochemical or flow mediated reasons. The mammary arteries are not the optimal control compared to non-diseased arteries prone to develop atherosclerosis. However, availability of non-diseased carotid artery is a drawback.

We did not execute a specific fat stain. However, the fat content of the plaques was clearly visible in the used HE stainings.

In summary, we show that levels of plaque EDA in human atherosclerotic lesions are associated with stable plaque phenotypes probably due to the higher number of smooth muscle cells that are the potential producers of human plaque EDA. This observation implies that the detection of endogenous Tlr ligand not necessarily represent an inflammatory environment destabilizing the vulnerable plaque, but also may trigger the stabilization of atherosclerotic lesions.

This is an observational study and therefore inferences regarding causality cannot be made. However, considering previous results from our own laboratory (unpublished data) and from Tan et al. [23] we feel that our data support the concept that EDA is actively involved in atherogenesis and should not be considered an innocent bystander.
No differences were found in plasma levels of EDA between groups investigated, suggesting that EDA in plasma cannot be used as diagnostic or prognostic plasma marker for atherosclerosis. The difference in EDA levels between stable and vulnerable plaque phenotypes identify EDA as a potential local plaque marker to image atherosclerotic plaques.

\section{Acknowledgements}

This study is supported by the Dutch Heart Foundation (project 2001-162, M93.001). We would like to thank Pleunie van den Borne for technical assistance.

\section{Appendix A. Supplementary data}

Supplementary data associated with this article can be found, in the online version, at doi:10.1016/j.atherosclerosis.2007.03.014.

\section{References}

[1] Hynes RO. Fibronectins. In: Fibronectins. New York: Springer-Verlag; 1990.

[2] Brown LF, Dubin D, Lavigne L, et al. Macrophages and fibroblasts express embryonic fibronectins during cutaneous wound healing. Am J Pathol 1993;142(March):793-801.

[3] Ffrench-Constant C, Van De WL, Dvorak HF, Hynes RO. Reappearance of an embryonic pattern of fibronectin splicing during wound healing in the adult rat. J Cell Biol 1989;109(August):903-14.

[4] Van Der Straaten HM, Canninga-van Dijk M, Verdonck LF, et al. Extradomain-A fibronectin: a new marker of fibrosis in cutaneous graftversus-host disease. J Invest Dermatol 2004;123(December):1057-62.

[5] Barnes JL, Hastings RR, De la Garza MA. Sequential expression of cellular fibronectin by platelets, macrophages, and mesangial cells in proliferative glomerulonephritis. Am J Pathol 1994;145(September):585-97.

[6] Jarnagin WR, Rockey DC, Koteliansky VE, Wang SS, Bissell DM. Expression of variant fibronectins in wound healing: cellular source and biological activity of the EIIIA segment in rat hepatic fibrogenesis. J Cell Biol 1994;127(December):2037-48.

[7] Kuhn C3, Boldt J, King TEJ, et al. An immunohistochemical study of architectural remodeling and connective tissue synthesis in pulmonary fibrosis. Am Rev Respir Dis 1989;140(December):1693-703.

[8] Kanters SD, Banga JD, Algra A, et al. Plasma levels of cellular fibronectin in diabetes. Diabetes Care 2001;24(February):323-7.

[9] Peters JH, Carsons S, Kalunian K, et al. Preferential recognition of a fragment species of osteoarthritic synovial fluid fibronectin by antibodies to the alternatively spliced EIIIA segment. Arthritis Rheum 2001;44(November):2572-85.

[10] Coito AJ, Brown LF, Peters JH, Kupiec-Weglinski JW, Van De Water L. Expression of fibronectin splicing variants in organ transplantation: a differential pattern between rat cardiac allografts and isografts. Am J Pathol 1997;150(May):1757-72.

[11] Dubin D, Peters JH, Brown LF, et al. Balloon catheterization induced arterial expression of embryonic fibronectins. Arterioscler Thromb Vasc Biol 1995;15(November):1958-67.

[12] Glukhova MA, Frid MG, Shekhonin BV, et al. Expression of extra domain A fibronectin sequence in vascular smooth muscle cells is phenotype dependent. J Cell Biol 1989;109(July):357-66. 
[13] Takasaki I, Chobanian AV, Mamuya WS, Brecher P. Hypertension induces alternatively spliced forms of fibronectin in rat aorta. Hypertension 1992;20(July):20-5.

[14] Okamura Y, Watari M, Jerud ES, et al. The extra domain A of fibronectin activates Toll-like receptor 4. J Biol Chem 2001;276(March):10229-33.

[15] Poltorak A, He X, Smirnova I, et al. Defective LPS signaling in $\mathrm{C} 3 \mathrm{H} / \mathrm{HeJ}$ and $\mathrm{C} 57 \mathrm{BL} / 10 \mathrm{ScCr}$ mice: mutations in Tlr4 gene. Science 1998;282(December):2085-8.

[16] Ohashi K, Burkart V, Flohe S, Kolb H. Cutting edge: heat shock protein 60 is a putative endogenous ligand of the toll-like receptor-4 complex. J Immunol 2000;164(January):558-61.

[17] Xu XH, Shah PK, Faure E, et al. Toll-like receptor-4 is expressed by macrophages in murine and human lipid-rich atherosclerotic plaques and upregulated by oxidized LDL. Circulation 2001;104(December):3103-8.

[18] Hollestelle SC, De Vries MR, Van Keulen JK, et al. Toll-like receptor 4 is involved in outward arterial remodeling. Circulation 2004;109(January):393-8.

[19] Michelsen KS, Wong MH, Shah PK, et al. Lack of Toll-like receptor 4 or myeloid differentiation factor 88 reduces atherosclerosis and alters plaque phenotype in mice deficient in apolipoprotein E. Proc Natl Acad Sci USA 2004;101(July (29)):10679-84.

[20] Pasterkamp G, Galis ZS, De Kleijn DP. Expansive arterial remodeling: location, location, location. Arterioscler Thromb Vasc Biol 2004;24(April (4)):650-7.

[21] Vink A, Schoneveld AH, Van Der Meer JJ, et al. In vivo evidence for a role of toll-like receptor 4 in the development of intimal lesions. Circulation 2002;106(October):1985-90.

[22] Wright SD, Burton C, Hernandez M, et al. Infectious agents are not necessary for murine atherogenesis. J Exp Med 2000;(April):1437-42.

[23] Tan MH, Sun Z, Opitz SL, et al. Deletion of the alternatively spliced fibronectin EIIIA domain in mice reduces atherosclerosis. Blood 2004;1(July (104)):11-8.

[24] Verhoeven BA, Velema E, Schoneveld AH, et al. Athero-express: differential atherosclerotic plaque expression of mRNA and protein in relation to cardiovascular events and patient characteristics. Rationale and design. Eur J Epidemiol 2004;19:1127-33.

[25] Simons PC, Algra A, van de Laak MF, Grobbee DE, Van Der GY. Second manifestations of ARTerial disease (SMART) study: rationale and design. Eur J Epidemiol 1999;15(October):773-81.

[26] Peters JH, Maunder RJ, Woolf AD, Cochrane CG, Ginsberg MH. Elevated plasma levels of ED1+ ("cellular") fibronectin in patients with vascular injury. J Lab Clin Med 1989;113(May (5)):58697.

[27] Sluijter JP, Pulskens WP, Schoneveld AH, et al. Matrix metalloproteinase 2 is associated with stable and matrix metalloproteinases 8 and 9 with vulnerable carotid atherosclerotic lesions: a study in human endarterectomy specimen pointing to a role for different extracellular matrix metalloproteinase inducer glycosylation forms. Stroke 2006;37(January (1)):235-9.

[28] Verhoeven B, Hellings WE, Moll FL, et al. Carotid atherosclerotic plaques in patients with transient ischemic attacks and stroke have unstable characteristics compared with plaques in asymptomatic and amaurosis fugax patients. J Vasc Surg 2005;42(December (6)):1075-81

[29] Singh P, Reimer CL, Peters JH, et al. The spatial and temporal expression patterns of integrin alpha9beta1 and one of its ligands, the EIIIA segment of fibronectin, in cutaneous wound healing. J Invest Dermatol 2004;123(December):1176-81.

[30] Sasu S, LaVerda D, Qureshi N, Golenbock DT, Beasley D. Chlamydia pneumoniae and chlamydial heat shock protein 60 stimulate proliferation of human vascular smooth muscle cells via toll-like receptor 4 and p44/p42 mitogen-activated protein kinase activation. Circ Res 2001;89(August):244-50.

[31] Edfeldt K, Swedenborg J, Hansson GK, Yan ZQ. Expression of tolllike receptors in human atherosclerotic lesions: a possible pathway for plaque activation. Circulation 2002;105(March):1158-61.

[32] Strauss BH, Chisholm RJ, Keeley FW, et al. Extracellular matrix remodeling after balloon angioplasty injury in a rabbit model of restenosis. Circ Res 1994;75(October (4)):650-8. 